\title{
ON-CHIP OPTOFLUIDIC RING RESONATOR SENSOR FOR MICRO-SCALE GAS CHROMATOGRAPHY
}

\author{
Kee Scholten, ${ }^{1,5}$ Xudong Fan, ${ }^{2,5}$ and Edward Zellers ${ }^{1,3-5^{*}}$ \\ ${ }^{1}$ Applied Physics Program, University of Michigan, Ann Arbor, MI, USA 48109-1040 \\ ${ }^{2}$ Department of Biomedical Engineering, University of Michigan, Ann Arbor, MI, 48109- 2110 \\ ${ }^{3}$ Department of Environmental Health Sciences, Univ. of Michigan, Ann Arbor, MI, 48109-2029 \\ ${ }^{4}$ Department of Chemistry, University of Michigan, Ann Arbor, MI, 48109-1055 \\ ${ }^{5}$ Center for Wireless Integrated MicroSensing and Systems, Univ. of Michigan, Ann Arbor, MI, 48109-2121
}

\begin{abstract}
The design, fabrication and preliminary performance assessment of a new vapor sensor comprising a microfabricated optofluidic ring resonator ( $\mu \mathrm{OFRR})$ and its application to microscale gas chromatographic $(\mu \mathrm{GC})$ detection of volatile organic compounds (VOC) are presented. The $\mu$ OFRR combines vapor sensing and fluidic transport functions in a micro-fabricated whispering gallery mode (WGM) resonator. The device also integrates on-chip fluidic-interconnection and fiber-optic probe alignment features. We demonstrate sub-second, sub-ng VOC detection and also couple the $\mu \mathrm{OFRR}$ to an upstream $\mu \mathrm{GC}$ column for rapid analysis of a simple VOC mixture.
\end{abstract}

\section{INTRODUCTION}

Advances in photonics have yielded a new class of (bio)chemical sensors adapted from WGM resonators [1], wherein shifts in resonant frequencies ( $\lambda$ WGM) reflect analyte interactions with a sensitive interface layer. The optofluidic ring resonator (OFRR) merges sensing and fluidic transport into a single structure [2]: the evanescent components of WGMs, excited in the wall of a dielectric capillary by an external waveguide, extend into the interior of the OFRR, and changes in RI at the inner surface can cause a shift in $\lambda$ wGM. Previous iterations include heat-drawn glass capillaries with sorptive-polymer films [3,4], and self-rolled microtubes of semiconductor multi-layers [5]. Yet, devices reported to date have not had the small size, durability, precision fabrication, high Q-factors, and fluidic compatibility for integration into $\mu \mathrm{GC}$ systems suitable for multi-VOC analyses in environmental or clinical applications.

Here we unveil a $1^{\text {st }}$-generation $\mu$ OFRR vapor sensor that improves upon prior OFRR designs and performs as well as, or better than, other microsensors used as $\mathrm{GC} / \mu \mathrm{GC}$ detectors [6-8]. Our device integrates a PDMS-coated, $\mathrm{SiO}_{\mathrm{x}} \mu \mathrm{OFRR}$ cylinder $(250-\mu \mathrm{m}$ i.d., $1.2-\mu \mathrm{m}$ wall; $85-\mu \mathrm{m}$ tall), a microfluidic interconnection channel, capillary insertion port, and an optical-fiber alignment structure on a $2 \times 2-\mathrm{cm} \mathrm{Si} \mathrm{chip} \mathrm{(Fig.} \mathrm{1).} \mathrm{High-Q}\left(>10^{4}\right)$ WGM resonances are excited in a centrally located quasi-toroidal modeconfinement expansion in the $\mu$ OFRR by a $1550-\mathrm{nm}$ laser via a fiber waveguide. This is a refinement of $\mu$ OFRR test structures we have made [9], which lacked a complete fluidic pathway.

\section{EXPERIMENTAL \\ $\mu$ OFRR Fabrication}

The $\mu$ OFRR sensor was micromachined from Si. The resonator cylinder pre-form was made by through-wafer DRIE. An interim isotropic $\mathrm{XeF}_{2}$ etch created a quasi-toroidal midsection expansion for mode confinement. Sidewalls were oxidized and HF-etched to reduce surface roughness before a final thermal oxidation step created the $\mathrm{SiO}_{x} \mu \mathrm{OFRR}$. The resonator was then partially released with a $\mathrm{XeF}_{2}$ etch to a height of $85 \mu \mathrm{m}$.
Backside DRIE was used to create a tapered trench for capillary insertion, and a microfluidic channel connecting the capillary port and backside $\mu$ OFRR aperture. A final frontside DRIE step created an optical-fiber alignment channel running laterally across the surface. Devices were diced into $2 \times 2 \mathrm{~cm}$ chips.

An internal PDMS layer was deposited in the $\mu$ OFRR by filling the resonator cavity with a toluene solution of PDMS and evaporating the solvent. The backside fluidic channels were sealed with a $2 \times 2 \mathrm{~cm}$ Pyrex coverplate using UV curable glue. A short section of fused-silica capillary $(250 \mu \mathrm{m}$ i.d.) was sealed into the completed device for external fluidic connection.

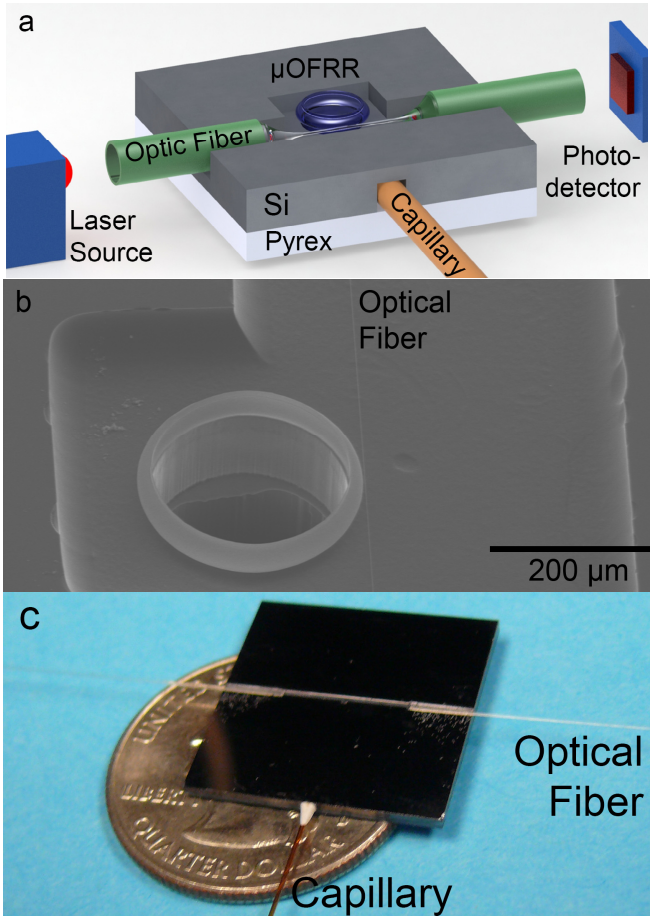

Fig. 1: a, Concept drawing of the $\mu O F R R$ sensor configuration. $b$, $S E M$ of $\mu$ OFRR with tapered optical fiber (right) in contact with the quasi-toroidal expansion. $c$, Photo of the $\mu O F R R$ sensor chip.

\section{MOFRR Sensor Tests}

WGM resonances were excited in the $\mu$ OFRR wall by evanescently coupling a laser signal from a thinned optical fiber $(\sim 1$ $\mu \mathrm{m}$ o.d.), placed in contact with the $\mu$ OFRR expansion. The fiber was connected to a tunable $1550 \mathrm{~nm}$ laser source and an IR photoreciever. The laser wavelength was swept over a $375 \mathrm{pm}$ range at $10 \mathrm{~Hz}$ while the transmitted signal was recorded.

The WGM was evident as a Lorentzian trough in the transmitted intensity. $\lambda$ wGM was defined as wavelength of minimum transmission and sensor response was defined as a shift in $\lambda_{\text {WGM }}$ due to VOC exposure. Test atmospheres of benzene, toluene, 
ethylbenzene, m-xylene, and n-octane were created in Tedlar bags at 5 concentrations. VOC aliquots were collected in a $1-\mathrm{mL}$ sample loop and injected into the $\mu$ OFRR in dry air at $3 \mathrm{~mL} / \mathrm{min}$. This was repeated with m-xylene using a $5-\mu \mathrm{L}$ sample loop to evaluate responses to transient exposures.

For $\mu \mathrm{GC}$ integration tests, the $\mu$ OFRR was connected downstream from a $3 \times 3 \mathrm{~cm}$ chip containing a $3-\mathrm{m}$ long PDMScoated DRIE separation $\mu$ column. A 4-VOC mixture was injected (5- $\mu \mathrm{L}$ loop) into the $\mu$ column in dry air at $1.4 \mathrm{~mL} / \mathrm{min}$.

\section{RESULTS AND DISCUSSIONS}

All VOC exposures induced reversible red shifts in $\lambda_{\text {WGM, }}$ with rapid steady-state responses for all VOCs (rise and fall times $<2.5$ $\mathrm{s}$ and $<5.8 \mathrm{~s}$, respectively). Fig. 2 presents calibration curves for the five individual vapors from steady-state responses. Sensitivity was defined as the slope of the calib. curve in $\mathrm{pm} /\left(\mathrm{mg} / \mathrm{m}^{3}\right)$, and limit of detection (LOD) was defined as $3 \sigma$ /sensitivity, where $\sigma$ is the std. deviation of the baseline. LODs ranged from 2.2 (benzene) to 22 $\mathrm{mg} / \mathrm{m}^{3}$ (ethylbenzene). The high sensitivity is attributed, in part, to the thin walls of the $\mu$ OFRR, which increase the portion of the WGM probing the polymer film.

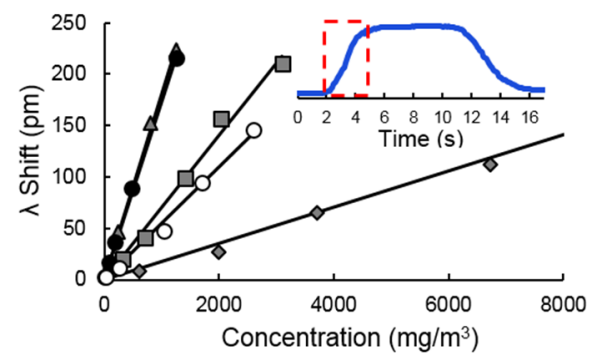

Fig. 2: Calibration curves for benzene (diamond), toluene (square), ethylbenzene (triangle), $m$-xylene (filled circle), and $n$ octane (unfilled circle) vapors. Inset shows the response profile to $700 \mathrm{mg} / \mathrm{m}^{3}$ of toluene, while red square shows $<2.5$ s rise time.

For transient exposures, peak height and peak area varied linearly $\left(\mathrm{R}^{2}>0.997\right)$ with injected mass. The calculated LOD of 49 pg is $10-1,000 \times$ lower than those of other microsensors adapted as $\mu \mathrm{GC}$ detectors [6,7] and $\sim 100 \times$ lower than a polymer-coated capillary-based OFRR [4]. Fig. 3 shows the $\mu$ OFRR response profile for 180-pg injection of m-xylene (FWHM $=0.71 \mathrm{~s})$ compared to a similar injection for a commercial FID under the same conditions (FWHM $=0.60 \mathrm{~s})$. This demonstrates the very low effective dead volume of the $\mu$ OFRR which, combined with its rapid response time, is critical for a high-speed $\mu \mathrm{GC}$ detector.

Fig. 4 shows the separation/detection of four VOCs with a $\mu \mathrm{GC}$ column chip coupled to the $\mu$ OFRR sensor. VOCs are easily separated in $<40 \mathrm{~s}$ and peaks are symmetric and sharp (FWHM = 0.33-1.17 s), reflecting excellent sensor response dynamics.

\section{CONCLUSIONS}

This is the first report of a microfabricated OFRR sensor and the first report of an on-chip optofluidic resonator for VOC sensing. Sensitivity is outstanding and response time is sufficient to capture very narrow peaks containing sub-ng quantities of vapor. This design reduces the size, increases structural integrity, and grants precise control of resonator dimensions, allowing for thinner walls and increased sensitivity, when compared to capillary drawn OFRRs. This is also the first report of an optofluidic resonator used to together with a $\mu \mathrm{GC}$ column. Results augur well for integration of the $\mu$ OFRRs into $\mu \mathrm{GC}$ systems.

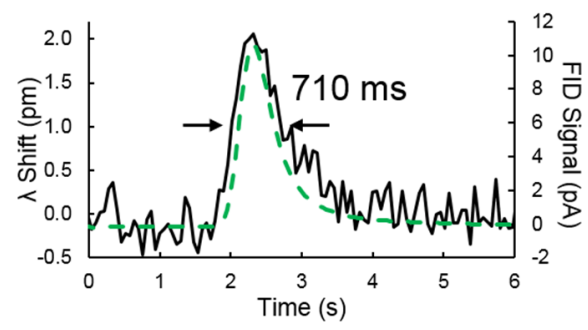

Fig. 3: Response profiles from the $\mu$ OFRR (solid black line, left axis) for a 180-pg injection of m-xylene and the FID (dashed green line, right axis) for a 500-pg injection of m-xylene; scaling of $y$ axes facilitate comparison of full-width-at-half-maximum (FWHM).

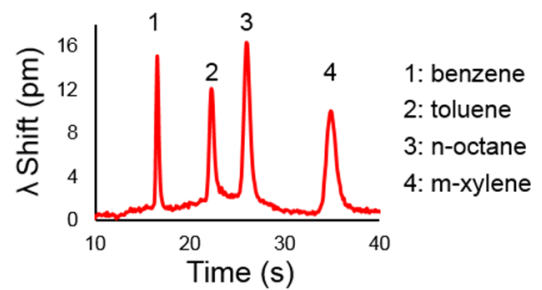

Fig. 4: Separation of benzene, toluene, $n$-octane and $m$-xylene using a pcolumn chip containing a 3-m long PDMS-coated channel at $63^{\circ} \mathrm{C}$ and the (downstream) $\mu$ OFRR sensor at $22^{\circ} \mathrm{C}$.

\section{ACKNOWLEDGMENTS}

We thank Mr. Will Collin for critical technical assistance with GC and $\mu \mathrm{GC}$ tests. This work was supported by NSF Grant ECCS 1128157. Devices were fabricated in the Lurie Nanofabrication Facility, a member of the NNIN supported by the NSF.

\section{REFERENCES}

[1] F. Vollmer, S. Arnold, "Whispering-Gallery-mode Biosensing: Label Free Detection Down to Single Molecules" Nat. Meth., 5, 591 (2008).

[2] X. Fan, I. White, "Optofluidic Microsystems for Chemical and Biological Analysis" Nat. Photon, 5, 591 (2011).

[3] Y. Sun, S.I. Shopova, G. Frye-Mason, X. Fan, "Rapid Chemical-Vapor Sensing Using Optofluidic Ring Resonators" Optics Lett., 33, 788 (2008).

[4] S. Shopova, et. al, "On-Column micro-GC Detection with Capillary-Based Optical Ring Resonators" Anal. Chem., 80, 2232 (2008).

[5] S.M. Harazim, et. al, "Lab-in-a-Tube: On-Chip Integration of Glass Optofluidic Ring Resonators for Label-Free Sensing Applications" Lab Chip, 12, 2649 (2012).

[6] M. Li., et. al, M. Roukes, "Nanoelectromechanical Resonator Arrays for Ultrafast, Gas-Phase Chromatographic Chemical Analysis" Nano Lett, 10, 3899 (2010).

[7] W. Collin, G. Serrano, L. Wright, H. Chang, N. Nunovero, E. T. Zellers, "Microfabricated Gas Chromatograph for Rapid, Trace-Level Determinations of Gas-Phase Explosive Marker Compounds" Anal. Chem., 86, 655 (2014).

[8] S. Zampolli, et. al, "Real-Time Monitoring of Sub-ppb Concentrations of Aromatic Volatiles with a MEMS-Enabled Gas Chromatograph," Sens. Act. B - Chem., 141, 322 (2009).

[9] K. Scholten, X. Fan, E.T. Zellers, "Microfabricated Optofluidic Ring Resonator Structures" App. Phys. Lett.,99, 141108 (2011).

\section{CONTACT}

E. T. Zellers, tel: +1-734-936-0766; ezellers@umich.edu 